\title{
Neue Serie: Translationale Forschung in der Pneumologie
}

\author{
New Series: Translational Research in Pneumology
}

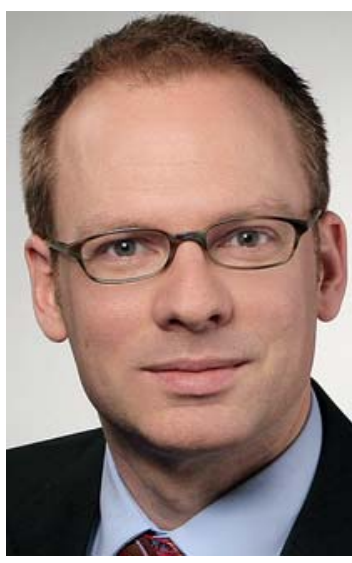

Prof. Dr. med. M. Witzenrath.
Pneumologische Erkrankungen verursachen hohe Mortalität, Morbidität und Kosten. Die meisten etablierten Therapien insbesondere chronischer und schwerer pneumologischer Erkrankungen lindern, ohne zu heilen. Deshalb werden neue, kurative Therapien benötigt, ebenso wie verbesserte Optionen für Diagnose und Prävention. Vor diesem Hintergrund ist die positive Entwicklung der pneumologischen Forschung weltweit und besonders in Deutschland während der letzten Jahrzehnte überaus erfreulich. Heute sind zahlreiche universitäre und außeruniversitäre Wissenschaftseinrichtungen aus dem deutschsprachigen Raum an der Erforschung von Lungenkrankheiten und der Entwicklung neuer pneumologischer Therapieansätze weltweit führend beteiligt.

Bereits in der jüngeren Vergangenheit hat das erweiterte Verständnis der Pathophysiologie verschiedener pneumologischer Erkrankungen zur klinischen Etablierung neuer Diagnose- und Therapiestrategien geführt. Erfreulicherweise deuten zahlreiche gegenwärtige präklinische Entwicklungen darauf hin, dass in naher Zukunft weitere, verbesserte Therapien den Sprung in die Klinik schaffen werden. Diese neuen diagnostischen, therapeutischen und präventiven Perspektiven entstehen ganz wesentlich durch den engen Dia$\log$ zwischen Grundlagenforschern und klinischen Wissenschaftlern, die "from bench to bedside“ und ebenso „from bedside to bench“ Erkenntnisse und Informationen austauschen und somit „Translationale Forschung“ lebendig machen. In einer neuen Artikelserie mit dem Titel
„Translationale Forschung in der Pneumologie“ widmet sich die Pneumologie aktuellen Errungenschaften mit hohem klinischen Potenzial. Namhafte Autoren aus deutschsprachigen Wissenschaftseinrichtungen werden über neue Entwicklungen aus der präklinischen und frühen klinischen Entwicklung berichten. So wird zunächst zweimonatlich je ein Artikel zu den Themen COPD (B. Schmeck, Marburg und R. Bals, Homburg/Saar), Asthma bronchiale (H. Fehrenbach, Borstel), Pneumonie (M. Witzenrath, Berlin), Interstitielle Pneumonie (A. Günther, Gießen), Sarkoidose (J. Müller-Quernheim, Freiburg), Mykobakterielle Infektionen (P. Zabel, Borstel), Pulmonale Hypertonie (A. und $\mathrm{H}$. Olschewski, Graz), Akutes Lungenversagen (H. Müller-Redetzky, Berlin) und Lungenbeteiligung bei rheumatischen $\mathrm{Er}$ krankungen (G. Riemekasten, Lübeck) erscheinen. Weitere Artikel sind in Planung.

Ziel dieser Artikelserie ist, pathophysiologische Zusammenhänge und damit die Rationale für neue Entwicklungen in Diagnostik, Prävention und Therapie zu verdeutlichen. Ferner sollen Neuentwicklungen unabhängig beschrieben und bewertet werden.

Wir hoffen, mit dieser neuen Serie den Leserinnen und Lesern der Pneumologie spannende Einblicke in die pneumologische Forschung und einen realistischen Ausblick in die nahe klinische Zukunft zu vermitteln.
Bibliografie

Dol http://dx.doi.org/

10.1055/s-0035-1563782

Pneumologie 2015; 69: 703

(c) Georg Thieme Verlag KG

Stuttgart · New York

ISSN 0934-8387

Korrespondenzadresse

Prof. Dr. med.

Martin Witzenrath

Medizinische Klinik mit

Schwerpunkt Infektiologie und

Pneumologie

Charité - Universitätsmedizin

Berlin

Charitéplatz 1

10117 Berlin

Martin.Witzenrath@charite.de 